\title{
Patient-Reported Symptoms Over 48 Weeks Among Participants in Randomized, Double-Blind, Phase III Non-inferiority Trials of Adults with HIV on Co-formulated Bictegravir, Emtricitabine, and Tenofovir Alafenamide versus Co-formulated Abacavir, Dolutegravir, and Lamivudine
}

\author{
David Wohl ${ }^{1}$ Amanda Clarke ${ }^{2} \cdot$ Franco Maggiolo $^{3} \cdot$ Will Garner $^{4} \cdot$ Marianne Laouri $^{4} \cdot$ Hal Martin $^{4} \cdot$ Erin Quirk $^{4}$
}

Published online: 29 June 2018

(c) The Author(s) 2018

\begin{abstract}
Background Integrase strand transfer inhibitors (INSTIs) are recommended for first-line antiretroviral therapy in combination with two nucleos(t)ide reverse transcriptase inhibitors. Co-formulated bictegravir, emtricitabine, and tenofovir alafenamide (B/F/TAF), a novel, INSTI-based regimen, is currently approved in the US and EU for the treatment of HIV-1 infection and recommended as first-line treatment in current guidelines. In our current analysis, we aimed to determine changes in patient-reported symptoms over time among HIV-1-infected adults who initiated or switched to B/F/TAF versus another INSTI-based regimen, co-formulated abacavir, dolutegravir, and lamivudine (ABC/DTG/3TC).

Methods A planned secondary analysis of patient-reported outcomes was conducted for two double-blind, randomized, phase III studies in HIV-1-infected adults comparing B/F/TAF with ABC/DTG/3TC: one in treatment-naïve individuals (GS-US-380-1489, ClinicalTrials.gov NCT02607930) and the other in virologically suppressed participants (GS-US-3801844, ClinicalTrials.gov NCT02603120). In both studies, the HIV symptoms distress module (HIV-SI) was administered at baseline (BL) and weeks 4, 12, and 48. Responses to each of the 20 items were dichotomized as bothersome or not bothersome. Treatment differences were assessed using unadjusted and adjusted logistic regression models (adjusted for BL HIV-SI count, age, sex, BL Veterans Aging Cohort Study [VACS] Index, medical history of serious mental illness, BL Short Form [SF]-36 Physical Component Summary [PCS], BL SF-36 Mental Component Summary [MCS], and, for virologically suppressed participants only, years since HIV diagnosis). We conducted longitudinal modeling of bothersome symptoms using a generalized mixed model including treatment, time, time-by-treatment, and additional covariates from the adjusted logistic regression model as described above. The Pittsburgh Sleep Quality Index (PSQI) was administered at the same frequency as the HIV-SI, and the total score was dichotomized as good or poor sleep quality. Similar models to those used for HIV-SI were applied, using BL sleep quality and BL SF-36 MCS as covariates. Statistical significance was assessed using $p<0.05$. Results Across both studies, bothersome symptoms were reported by fewer participants on B/F/TAF than those on ABC/ DTG/3TC. In treatment-naïve adults, fatigue/loss of energy, nausea/vomiting, dizzy/lightheadedness, and difficulty sleeping were reported significantly less with B/F/TAF at two or more time points. Fatigue and nausea were also significantly less common for those receiving B/F/TAF in longitudinal models. In virologically suppressed participants, nausea/vomiting, sad/ down/depressed, nervous/anxious, and poor sleep quality (from the PSQI) were reported significantly less with B/F/TAF at two or more time points, as well as in longitudinal models.
\end{abstract}

Conclusions B/F/TAF was associated with lower prevalence of bothersome symptoms than ABC/DTG/3TC in both treatmentnaïve and virologically suppressed adults.

Electronic supplementary material The online version of this article (https://doi.org/10.1007/s40271-018-0322-8) contains supplementary material, which is available to authorized users.

Extended author information available on the last page of the article 


\section{Key points for decision makers}

As the efficacy of triple-therapy antiretroviral regimens remains consistently high, patient well-being (e.g., patient-reported outcomes [PROs]) has become an important differentiator between regimens.

We evaluated PROs in two studies comparing coformulated bictegravir, emtricitabine, and tenofovir alafenamide (B/F/TAF) with co-formulated abacavir, dolutegravir, and lamivudine (ABC/DTG/3TC), where treatment differences were noted if prevalence was statistically significantly different at two or more time points in the adjusted logistic regression model or at one time point in the adjusted logistic regression model and in the longitudinal model.

Among treatment-naïve participants, initiating B/F/ TAF was associated with lower prevalence of fatigue/ loss of energy, dizzy/lightheadedness, nausea/vomiting, difficulty sleeping, and loss of appetite compared with $\mathrm{ABC} / \mathrm{DTG} / 3 \mathrm{TC}$.

For virologically suppressed participants, switching to $\mathrm{B} / \mathrm{F} / \mathrm{TAF}$ was associated with lower prevalence of dizzy/ lightheadedness, nausea/vomiting, sad/down/depressed, nervous/anxious, difficulty sleeping, and loss of appetite compared with remaining on ABC/DTG/3TC.

In both patient populations, no symptom had a greater prevalence with $\mathrm{B} / \mathrm{F} / \mathrm{TAF}$ compared with $\mathrm{ABC} /$

DTG/3TC.

\section{Introduction}

Over the past decade, antiretroviral treatments for HIV infection have demonstrated high potency, yielding viral suppression rates above $90 \%$. Integrase strand inhibitors (INSTIs; e.g., bictegravir, dolutegravir, elvitegravir, and raltegravir) in combination with two nucleos(t)ide reverse transcriptase inhibitors (NRTIs) are currently recommended as initial treatment for HIV in many international guidelines [1-3]. As the success of antiretroviral therapy largely depends on patient adherence, fixed-dose combinations (FDCs) have emerged as preferred treatment regimens and are associated with less treatment failure [4]. The impact of FDCs on an individual's overall health, including sense of wellbeing, has also become an important consideration for clinicians when prescribing HIV treatment.

Bictegravir (BIC, B) and dolutegravir (DTG) are INSTIs that do not require the co-administration of a pharmacoenhancer (i.e., cobicistat or ritonavir) [5]. Both have been co-formulated with NRTIs as FDCs: BIC with emtricitabine (FTC, F) and tenofovir alafenamide (TAF) as B/F/ TAF and DTG with abacavir (ABC) and lamivudine (3TC) as $\mathrm{ABC} / \mathrm{DTG} / 3 \mathrm{TC}$. ABC/DTG/3TC is currently recommended as first-line antiretroviral therapy (ART) [1-3] and represents a logical, standard-of-care comparator for new INSTI-based FDCs. ABC requires HLA B*5701 testing prior to use, and the $\mathrm{ABC} / \mathrm{DTG} / 3 \mathrm{TC}$ co-formulation does not provide adequate treatment for hepatitis $\mathrm{B}$ in individuals co-infected with HIV [1,3]. The US AIDS Clinical Trials Group (ACTG) 5202 study found a significantly shorter time to treatment modification and a greater incidence of nausea with the ABC/3TC NRTI backbone than with FTC/ tenofovir disoproxil fumarate [6]. Furthermore, some reports have emerged showing associations between DTG and neuropsychiatric adverse events, while others have not [7-13].

BIC has a high barrier to resistance and a low potential for drug-drug interactions [14]. In four studies reported to date (two in treatment-naïve adults and two in virologically suppressed participants), the B/F/TAF FDC has shown similar high efficacy and tolerability to standard-of-care comparators, with no treatment-emergent resistance [15-18]. The primary outcome of all studies demonstrated non-inferiority of $\mathrm{B} / \mathrm{F} / \mathrm{TAF}$ to either ABC/DTG/3TC, DTG +F/TAF, or boosted protease-inhibitor (PI) regimens. Switching to $\mathrm{B} / \mathrm{F} /$ TAF from ABC/DTG/3TC specifically has the potential to maintain high rates of suppression while avoiding limitations of ABC, such as potential cardiovascular risk [19-21], as well as central nervous system adverse effects and discontinuations that have been reported more frequently with DTG in clinical practice and cohort studies than in published results of clinical trials $[9,22,23]$. $\mathrm{B} / \mathrm{F} / \mathrm{TAF}$ also provides two NtRTIs with hepatitis B activity and the tablet is less than half the size of co-formulated $\mathrm{ABC} / \mathrm{DTG} / 3 \mathrm{TC}$, which may improve acceptability amongst individuals and ability to use in pediatric populations [24-26, data on file]. B/F/ TAF has been approved in the US and EU for treatment of HIV-1 infection in adults and adolescents and is now also recommended as first-line ART in current treatment guidelines [3].

Health-related quality-of-life (HRQL) outcomes have long been regarded as important in the evaluation and differentiation of treatment strategies [27]. To better understand the two FDCs of B/F/TAF and ABC/DTG/3TC, patientreported outcomes (PROs), including one specifically designed for HIV-1-infected participants, were measured in both treatment-naïve and HIV-1-suppressed adults using previously validated questionnaires in comparative trials of these regimens. For treatment-naïve individuals, characterization of PROs provided the opportunity to assess the two regimens directly after initiating treatment. Comparison of the treatments in virologically suppressed individuals who were presumably tolerating their baseline regimen of $\mathrm{ABC} /$ 
DTG/3TC provided further delineation of how changes to medication may alter quality-of-life outcomes. We aimed to achieve a better understanding of the relationship between these treatments and bothersome HIV symptoms and/or adverse events that impact adherence and persistence.

\section{Methods}

\subsection{Study Design}

Study design and participant recruitment for both trials have been previously described [15, 17]. Briefly, both were international, double-blind, randomized, phase III studies that compared the efficacy and safety of B/F/TAF (50/200/25 mg) with ABC/DTG/3TC $(600 / 50 / 300 \mathrm{mg})$ in adults who were either treatment naïve (GS-US-380-1489, ClinicalTrials.gov NCT02607930) or virologically suppressed (GS-US-3801844, ClinicalTrials.gov NCT02603120). For both studies, post-baseline study visits occurred at weeks $4,8,12,24$, 36, and 48, with PRO measures administered at baseline and weeks 4, 12, and 48. Participants and investigators were masked to treatment allocation in both studies. Translated versions of all PRO tools were administered in the participant's preferred language.

\subsection{Baseline Demographics and Characteristics}

Demographics (sex, age, race, and ethnicity) and clinical characteristics (serious mental illness, CD4 cell count, asymptomatic HIV infection status, years since HIV diagnosis, years since first antiretroviral therapy use (GS-US380-1844 only), the Veterans Aging Cohort Study [VACS] Risk Index, and Fibrosis [FIB]-4 score) were collected or calculated. Serious mental illness was defined as a diagnostic history entered as existing medical history at the time of study entry of one or more of the following conditions based on medical chart review: major depression, anxiety, schizophrenia, bipolar disorder, post-traumatic stress disorder, or other serious mental illness. The VACS Index was calculated to quantify the overall mortality risk associated with HIV and is a summary score based on age, CD4 count, HIV-1 RNA, the FIB-4 score, creatinine, and coinfection with viral hepatitis $\mathrm{C}$ infection, and is used to predict all-cause and cause-specific mortality and other outcomes in those living with HIV infection [28]. The FIB-4 score, computed using age, platelet count, and aspartate and alanine transaminase values, provides an estimate of the degree of liver fibrosis in HIV and hepatitis C virus co-infected patients [29].

\subsection{Patient-Reported Outcomes (PROs)}

\subsubsection{HIV Symptom Distress Module (HIV-Symptom Index [HIV-SI])}

The dependent variable in this study was the HIV-Symptom Index (HIV-SI). The HIV-SI is a validated PRO instrument that assesses the burden of 20 common symptoms associated with HIV treatment or disease [30]. The instrument is considered to be the gold standard in contemporary HIV symptom research [31]. Respondents are asked about their experience with each of 20 symptoms during the past 4 weeks using a 5-point, Likert-type scale. Response options and scores are as follows: (0) "I don't have this symptom;" (1) "I have this symptom and it doesn't bother me;" (2) "I have this symptom and it bothers me a little;" (3) "I have this symptom and it bothers me;" (4) "I have this symptom and it bothers me a lot."

The 20 symptoms comprising the HIV-SI are fatigue/ loss of energy, difficulty sleeping, nervous/anxious, diarrhea/loose bowels, changes in body composition, feeling $\mathrm{sad} /$ down/depressed, bloating/pain/gas in stomach, muscle aches/joint pain, problems with sex, trouble remembering, headaches, pain/numbness/tingling in hands/feet, skin problems/rash/itching, cough/trouble breathing, fever/chills/ sweats, dizzy/lightheadedness, weight loss/wasting, nausea/ vomiting, hair loss/changes, and loss of appetite/food taste.

Consistent with prior analyses by Edelman and colleagues [32], we dichotomized symptoms into not bothersome (scores of 0 or 1) or bothersome (scores of 2, 3 and 4). The overall bothersome symptom count at baseline was generated by counting the number of individual symptoms scored as bothersome and used as a covariate in regression analyses and longitudinal modeling.

\subsubsection{Other Descriptive PRO Measures}

Other PRO instruments used to describe HRQL included the Short Form (SF)-36, the Pittsburgh Sleep Quality Index (PSQI), and the Work Productivity and Activity Impairment (WPAI) - General Health questionnaire. The SF-36 is an instrument supported by extensive evidence of good psychometric properties in a range of therapeutic areas [33, 34], including HIV-infected individuals [35]. The score ranges from 0 to 100, with a higher score indicating better functioning. For our analyses, we further transformed the physical component summary (PCS) and mental component summary (MCS) scores into norm-based scoring, with a mean of 50 and a standard deviation of 10 using the QualityMetric Health Outcomes ${ }^{\mathrm{TM}}$ Scoring Software 4.5 (QualityMetric 
Incorporated [now part of OPTUM], Lincoln, RI, USA). The PSQI is a validated, 19-item instrument that assesses sleep quality and disturbances over a 1-month time interval; the total score is dichotomized into poor or good sleep quality [36]. The total score ranges from 0 to 21 , with a lower score indicating better sleep quality. The WPAI-General Health questionnaire is a 6-item tool that measures health-related, work productivity loss for the employed population as per the amount of absenteeism, presenteeism, and daily activity impairment attributable to general health [37]. For each of these measures, a lower value indicates better quality of life outcome. Additionally, the PCS and MCS from SF-36 were used as covariates in regression and longitudinal analyses of the HIV-SI.

\subsection{Statistical Analysis}

Analyses were performed using SAS v9.4M3 (SAS Institute, Cary, NC, USA). If multiple responses were provided for an item on the HIV-SI, the most severe (i.e., largest value) of the responses was used.

Baseline demographic and clinical characteristics were summarized using descriptive statistics. To determine between-treatment comparisons, we used the Fisher exact test for categorical data or a 2-sided Wilcoxon rank sum test for continuous data. The change from baseline in SF-36 PCS/MCS scores and WPAI scores were summarized using descriptive statistics, and a 2-sided Wilcoxon rank sum test was used for between-treatment comparisons. The prevalence (i.e., number and percentage of participants) of reported poor sleep quality on the PSQI was also summarized, and the Fisher exact test was used for betweentreatment comparison. Unadjusted and adjusted analyses at weeks 4,12 , and 48 were performed to evaluate the relationship between treatment assignment and the probability of experiencing an HIV-SI symptom, with and without additional covariates (adjusted and unadjusted, respectively). Specifically, HIV-SI symptoms were modeled as binary outcomes using a logistic regression. Descriptive variables were evaluated for multicollinearity and models were fitted/ tested with independent variables of treatment and a subset of clinical and demographic covariates (selected from a larger list of potential demographic and clinical variables). The subset of clinical and demographic covariates was selected in two steps. First, for each symptom/time point, stepwise model selection was used to identify statistically significant covariates from all potential covariates (i.e., all these baseline demographic and clinical characteristics listed in Table 1, baseline HIV-1 RNA, and baseline C4 cell counts). Second, all the sixty models (i.e., 20 symptoms $\times 3$ visits) were reviewed and a single set of covariates were selected across all models to ensure easy interpretation and simplification of the model. Furthermore, the same model was generally used for both studies, with the exception of utilizing years since HIV diagnosis in Study 380-1844 only.

Longitudinal modeling was performed using generalized mixed-effects models to show symptom patterns over each of the four study visits using data from the HIV-SI. The functional form of the change pattern was assessed visually from the observed prevalence in each group. Linear and quadratic patterns were tested to determine optimal fit, ultimately favoring a linear function. Post-baseline data were modeled with baseline values included as a covariate. To assess the possibility that the effect of treatment may itself vary over time, the models included an interaction between study treatment and time in addition to the indicator of a simple study treatment group. Continuous variables were mean centered for ease of interpretation and model fit. The fit of each of the derived models was compared with a simple unadjusted model that included time and study treatment, along with a random intercept to account for the longitudinal nature of the data. The comparison was based on Bayesian information criterion.

Poor sleep quality according to the PSQI was analyzed in a similar manner as the HIV-SI, but only used baseline sleep quality (good vs poor) and baseline SF-36 MCS as covariates.

\section{Results}

\subsection{Baseline Characteristics}

Baseline demographics and clinical characteristics were similar between treatment groups (Table 1) in each study. Most participants were White and male. Participants reported a median of three to four bothersome HIV-SI symptoms at baseline, with $17-25 \%$ reporting no symptoms. Overall, there were no significant differences at baseline between those assigned B/F/TAF compared with ABC/DTG/3TC in any PRO measure (Table 1). Among the items captured by the HIV-SI for both treatment-naïve and virologically suppressed adults, the only significant difference between treatment groups was more weight loss/wasting reported at baseline among those assigned $\mathrm{B} / \mathrm{F} / \mathrm{TAF}$ versus $\mathrm{ABC} / \mathrm{DTG} / 3 \mathrm{TC}$ (18.3 vs $11.3 \% ; p=0.017)$ in treatment-naive participants (study 380-1489) (Tables 2, 3).

\subsection{Changes in PRO Measures from Baseline to Week 48}

In treatment-naive adults (Study 380-1489), median [IQR] SF-36 PCS and MCS scores were comparable between the $\mathrm{B} / \mathrm{F} / \mathrm{TAF}$ and $\mathrm{ABC} / \mathrm{DTG} / 3 \mathrm{TC}$ groups at baseline (57.4 [52.6-60.0] vs 56.6 [52.2-59.3], $p=0.18$ and 49.0 
Table 1 Patient-reported baseline demographics and clinical characteristics

\begin{tabular}{|c|c|c|c|c|c|c|}
\hline & \multicolumn{3}{|c|}{ Study GS-US-380-1489 (treatment-naïve) } & \multicolumn{3}{|c|}{ Study GS-US-380-1844 (HIV-1 suppressed) } \\
\hline & $\begin{array}{l}\mathrm{B} / \mathrm{F} / \mathrm{TAF} \\
(N=314)\end{array}$ & $\begin{array}{l}\text { ABC/DTG/3TC } \\
(N=315)\end{array}$ & $p$ value & $\begin{array}{l}\mathrm{B} / \mathrm{F} / \mathrm{TAF} \\
(N=282)\end{array}$ & $\begin{array}{l}\mathrm{ABC} / \mathrm{DTG} / 3 \mathrm{TC} \\
(N=281)\end{array}$ & $p$ value \\
\hline Age (years) & $31(18-71)$ & $32(18-68)$ & 0.72 & $47(21-71)$ & $45(20-70)$ & 0.32 \\
\hline Male, $n(\%)$ & $285(90.8 \%)$ & $282(89.5 \%)$ & 0.69 & $247(87.6 \%)$ & $252(89.7 \%)$ & 0.51 \\
\hline Race, $n(\%)$ & & & 0.87 & & & 0.92 \\
\hline White & $180(57.7 \%)$ & $179(56.8 \%)$ & & $206(73.0 \%)$ & $202(72.7 \%)$ & \\
\hline Non-white & $132(42.3 \%)$ & $136(43.2 \%)$ & & $76(27.0 \%)$ & $76(27.3 \%)$ & \\
\hline Estimated GFR (mL/min) & $126(108-146)$ & $123(107-144)$ & 0.76 & $101(85-119)$ & $101(85-122)$ & 0.73 \\
\hline VACS Index score ${ }^{a}$ & $17(13-24)$ & $14(13-23)$ & 0.34 & $12(0-18)$ & $10(0-18)$ & 0.19 \\
\hline CD4 cell count (cells $/ \mu \mathrm{L})$ & $443(299-590)$ & $450(324-608)$ & 0.22 & $732(554-936)$ & $661(478-874)$ & 0.011 \\
\hline FIB-4 Index score ${ }^{b}$ & $0.8(0.6-1.1)$ & $0.7(0.6-1.1)$ & 0.75 & $0.9(0.7-1.2)$ & $0.9(0.7-1.2)$ & 0.78 \\
\hline Asymptomatic, $n(\%)$ & $286(91.1 \%)$ & $286(90.8 \%)$ & 1.00 & $243(86.2 \%)$ & $245(87.2 \%)$ & 0.80 \\
\hline Serious mental illness ${ }^{\mathrm{c}}, n(\%)$ & $91(29.0 \%)$ & $90(28.6 \%)$ & 0.93 & $122(43.3 \%)$ & $112(39.9 \%)$ & 0.44 \\
\hline HIV-SI symptom count $^{\mathrm{d}}$ & $4.0(1.0-7.0)$ & $3.0(1.0-7.0)$ & 0.44 & $3.0(1.0-6.5)$ & $3.0(1.0-5.0)$ & 0.48 \\
\hline Years since HIV diagnosis & $0.0(0.0-1.0)$ & $0.0(0.0-1.0)$ & 0.49 & $8.0(4.0-13.0)$ & $7.0(3.0-14.0)$ & 0.64 \\
\hline SF-36 PCS ${ }^{e}$ & $57.4(52.6-60.0)$ & $56.6(52.2-59.3)$ & 0.18 & $55.5(50.5-59.1)$ & $56.6(51.0-59.2)$ & 0.31 \\
\hline SF-36 $\mathrm{MCS}^{\mathrm{e}}$ & $49.0(37.7-55.2)$ & $49.5(40.0-56.3)$ & 0.40 & $51.9(44.5-57.5)$ & $53.2(46.6-57.6)$ & 0.14 \\
\hline
\end{tabular}

Data are reported as median (IQR) or $n(\%)$, except for age, which is median (range)

For categorical data, the $p$ value was from the Fisher exact test. For continuous data, $p$ value was from the 2-sided Wilcoxon rank sum test

Subjects with race of 'Not Permitted' were excluded from percentage calculations

$A B C / D T G / 3 T C$ co-formulated abacavir, dolutegravir, and lamivudine, $B / F / T A F$ co-formulated bictegravir, emtricitabine, and tenofovir alafenamide, FIB-4 Fibrosis 4 score, GFR glomerular filtration rate, HIV-SI HIV Symptom Index, SF-36 MCS Short Form 36 Mental Composite Score, SF-36 PCS Short Form 36 Physical Composite Score, VACS Veterans Aging Cohort Study

${ }^{a}$ VACS Index is a score from 0 to 164 derived from the patient's age, CD4 cell count, HIV-1 RNA level, hemoglobin value, platelet count, aspartate and alanine transaminase levels, serum creatinine value, and a positive hepatitis $\mathrm{C}$ infection status at one specific time point, with lower scores indicating better health

${ }^{\mathrm{b}}$ The FIB-4 score is derived from age, and platelets, aspartate and alanine transaminase values

${ }^{\mathrm{c}}$ Serious mental illness is defined as a medical history of at least one of the following diagnoses reported by the study investigator: major depression, anxiety, schizophrenia, bipolar disorder, post-traumatic stress disorder, or other psychosis

${ }^{\mathrm{d}}$ The HIV-SI bothersome symptom count is a summation of the presence of the individual HIV-SI items and ranges from 0 to 20 , with higher counts indicating more bothersome symptoms

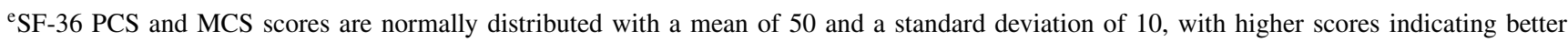
health

[37.7-55.2] vs 49.5 [40.0-56.3], $p=0.40$, respectively). Changes from baseline at week 48 in PCS and MCS scores were also similar between groups; median (IQR) change from baseline in PCS was 0.1 ( -3.3 to 3.1) versus $0.2(-2.6$ to 2.8 ), $p=0.85$ and in MCS was 2.3 ( -1.6 to 9.0 ) versus 2.1 ( -4.0 to 7.0$), p=0.090$. At baseline, 48.1 and $47.6 \%$ of participants on B/F/TAF and ABC/DTG/3TC, respectively, reported poor sleep quality on the PSQI $(p=0.93)$. At week 48 , the proportion had decreased to 38.7 and $41.5 \%$ of participants, respectively $(p=0.50)$. The WPAI questionnaire did not show any differences between treatment groups at baseline or any post-baseline time point.

In virologically suppressed adults (Study 380-1844), median [IQR] SF-36 PCS and MCS scores were comparable between the $\mathrm{B} / \mathrm{F} / \mathrm{TAF}$ and $\mathrm{ABC} / \mathrm{DTG} / 3 \mathrm{TC}$ groups at baseline (55.5 [50.5-59.1] vs 56.6 [51.0-59.2], $p=0.31$ and 51.9 [44.5-57.5] vs 53.2 [46.6-57.6], $p=0.14$, respectively). At week 48, median [IQR] PCS and MCS scores were largely unchanged from baseline (PCS change: $-0.4[-3.6$ to 2.7] vs 0.2 [ -2.3 to 2.7], $p=0.17$; MCS change: median [IQR]: 0.3 [ -3.0 to 4.6$]$ vs 0.1 [ -3.9 to 3.5$], p=0.13)$. At baseline, 51.9 and $46.1 \%$ of adults on B/F/TAF and ABC/ DTG/3TC, respectively, reported poor sleep quality on the PSQI $(p=0.19)$. At week 48 , the proportion had decreased to 41.8 and $45.7 \%$ of individuals, respectively $(p=0.42)$.

The WPAI questionnaire did not show any differences between treatment groups in any of the four measures at baseline or any post-baseline time point. 
Table 2 Frequency of bothersome HIV symptoms by treatment and study visit in Study GS-US-380-1489 (treatment-naïve)

\begin{tabular}{|c|c|c|c|c|c|c|c|c|}
\hline \multirow{2}{*}{$\begin{array}{l}\text { Individuals reporting } \\
\text { symptom, } \%\end{array}$} & \multicolumn{2}{|l|}{ Baseline } & \multicolumn{2}{|l|}{ Week 4} & \multicolumn{2}{|l|}{ Week 12} & \multicolumn{2}{|l|}{ Week 48} \\
\hline & $\begin{array}{l}\mathrm{B} / \mathrm{F} / \mathrm{TAF} \\
(n=311)\end{array}$ & $\begin{array}{l}\mathrm{ABC} / \mathrm{DTG} / 3 \mathrm{TC} \\
(n=313)\end{array}$ & $\begin{array}{l}\mathrm{B} / \mathrm{F} / \mathrm{TAF} \\
(n=313)\end{array}$ & $\begin{array}{l}\mathrm{ABC} / \mathrm{DTG} / 3 \mathrm{TC} \\
(n=310)\end{array}$ & $\begin{array}{l}\mathrm{B} / \mathrm{F} / \mathrm{TAF} \\
(n=307)\end{array}$ & $\begin{array}{l}\mathrm{ABC} / \mathrm{DTG} / 3 \mathrm{TC} \\
(n=309)\end{array}$ & $\begin{array}{l}\mathrm{B} / \mathrm{F} / \mathrm{TAF} \\
(n=293)\end{array}$ & $\begin{array}{l}\text { ABC/DTG/3TC } \\
(n=298)\end{array}$ \\
\hline Fatigue/loss of energy & 47.3 & 46.6 & 37.7 & 47.1 & 33.6 & 41.7 & 32.8 & 41.4 \\
\hline Fevers/chills/sweats & 26.7 & 21.7 & 20.4 & 18.7 & 14.0 & 15.5 & 15.4 & 14.8 \\
\hline Dizzy/lightheadedness & 21.3 & 17.7 & 17.6 & 23.5 & 15.0 & 17.6 & 13.0 & 21.5 \\
\hline $\begin{array}{l}\text { Pain/numbness/tingling } \\
\text { in hands/feet }\end{array}$ & 20.3 & 16.0 & 14.7 & 16.8 & 16.4 & 17.5 & 19.1 & 19.6 \\
\hline Trouble remembering & 22.8 & 18.0 & 20.1 & 19.0 & 23.8 & 20.4 & 20.8 & 21.5 \\
\hline Nausea/vomiting & 8.4 & 8.0 & 13.7 & 23.9 & 7.5 & 15.6 & 9.6 & 12.4 \\
\hline Diarrhea/loose bowels & 20.9 & 18.9 & 20.8 & 21.3 & 15.6 & 18.8 & 11.6 & 14.8 \\
\hline $\mathrm{Sad} /$ down/depressed & 41.9 & 36.5 & 31.9 & 35.5 & 33.2 & 32.4 & 27.6 & 31.3 \\
\hline Nervous/anxious & 45.3 & 38.0 & 31.9 & 32.9 & 27.8 & 30.1 & 28.8 & 30.2 \\
\hline Difficulty sleeping & 35.8 & 39.6 & 32.9 & 35.8 & 29.1 & 38.2 & 29.4 & 36.2 \\
\hline $\begin{array}{l}\text { Skin problems/rash/ } \\
\text { itching }\end{array}$ & 27.3 & 28.5 & 21.7 & 25.8 & 19.5 & 22.3 & 20.8 & 22.1 \\
\hline $\begin{array}{l}\text { Coughing/trouble } \\
\text { breathing }\end{array}$ & 15.4 & 15.4 & 13.4 & 14.0 & 14.4 & 13.9 & 12.7 & 14.1 \\
\hline Headaches & 21.9 & 21.2 & 20.8 & 22.7 & 18.6 & 21.0 & 13.0 & 22.9 \\
\hline Loss of appetite & 16.8 & 14.8 & 13.4 & 16.6 & 10.5 & 18.1 & 9.6 & 12.8 \\
\hline $\begin{array}{l}\text { Bloating/pain/gas in } \\
\text { stomach }\end{array}$ & 19.7 & 21.0 & 24.4 & 24.4 & 20.3 & 22.0 & 18.5 & 25.3 \\
\hline Muscle aches/joint pain & 27.1 & 28.3 & 19.2 & 21.1 & 23.6 & 22.3 & 21.6 & 25.3 \\
\hline Problems with sex & 26.1 & 26.4 & 18.6 & 17.2 & 18.0 & 16.2 & 17.5 & 18.9 \\
\hline $\begin{array}{l}\text { Changes in body com- } \\
\text { position }\end{array}$ & 17.4 & 15.2 & 14.7 & 12.3 & 17.4 & 15.2 & 19.5 & 22.9 \\
\hline Weight loss/wasting & 18.3 & 11.3 & 9.6 & 9.4 & 7.2 & 11.4 & 11.6 & 11.4 \\
\hline Hair loss/changes & 10.3 & 12.9 & 6.7 & 9.4 & 6.9 & 8.4 & 5.5 & 8.8 \\
\hline
\end{tabular}

$n$ is the number of participants with response for at least one symptom

Bold characters are for significantly different percentages between treatment groups $(p<0.05), p$ value is calculated from the unadjusted logistic regression model

$A B C / D T G / 3 T C$ co-formulated abacavir, dolutegravir, and lamivudine, $B / F / T A F$ co-formulated bictegravir, emtricitabine, and tenofovir alafenamide

\subsection{Associations Between HIV-SI Bothersome Symptoms and Treatment in Logistic Regression Models and Longitudinal Analyses}

The association between treatment and each bothersome symptom from the HIV-SI was examined by logistic regression models and longitudinal analyses. In the final models, treatment group (B/F/TAF vs ABC/DTG/3TC) was the independent variable and covariates included age, sex, race (White vs non-White), baseline bothersome symptom count, VACS Index score, medical history of serious mental illness (yes vs no), baseline SF-36 PCS, baseline SF-36 MCS, and years since HIV diagnosis (for the suppressed study only).

In treatment-naive participants, the adjusted logistic regression models showed that $\mathrm{B} / \mathrm{F} / \mathrm{TAF}$ was associated with a lower risk of experiencing four bothersome symptoms at two or more time points (three symptoms included week
48): fatigue/loss of energy, dizzy/lightheadedness, nausea/ vomiting, and difficulty sleeping. In virologically suppressed adults, the adjusted logistic regression models showed that $\mathrm{B} / \mathrm{F} / \mathrm{TAF}$ was associated with a lower risk of experiencing three symptoms at two or more time points (all symptoms included week 48): nausea/vomiting, sad/down/depressed, and nervous/anxious. A complete table of the unadjusted and adjusted logistic regression models appears as Table ESM1 in the Electronic Supplementary Material.

The prevalence of bothersome symptoms over time was evaluated using mixed-effects logistic models adjusted for the same covariates as specified above and also included time and time-by-treatment in the model. In treatmentnaïve adults, the adjusted longitudinal models revealed a statistically significant difference in the prevalence of three bothersome symptoms (fatigue/loss of energy, nausea/vomiting, and loss of appetite) between the $\mathrm{B} / \mathrm{F} / \mathrm{TAF}$ and $\mathrm{ABC} /$ 
Table 3 Frequency of bothersome HIV symptoms by treatment and study visit in Study GS-US-380-1844 (HIV-1 suppressed)

\begin{tabular}{|c|c|c|c|c|c|c|c|c|}
\hline \multirow{2}{*}{$\begin{array}{l}\text { Individuals reporting } \\
\text { symptom, } \%\end{array}$} & \multicolumn{2}{|l|}{ Baseline } & \multicolumn{2}{|l|}{ Week 4} & \multicolumn{2}{|l|}{ Week 12} & \multicolumn{2}{|l|}{ Week 48} \\
\hline & $\begin{array}{l}\mathrm{B} / \mathrm{F} / \mathrm{TAF} \\
(n=280)\end{array}$ & $\begin{array}{l}\mathrm{ABC} / \mathrm{DTG} / 3 \mathrm{TC} \\
(n=281)\end{array}$ & $\begin{array}{l}\mathrm{B} / \mathrm{F} / \mathrm{TAF} \\
(n=281)\end{array}$ & $\begin{array}{l}\mathrm{ABC} / \mathrm{DTG} / 3 \mathrm{TC} \\
(n=280)\end{array}$ & $\begin{array}{l}\mathrm{B} / \mathrm{F} / \mathrm{TAF} \\
(n=278)\end{array}$ & $\begin{array}{l}\mathrm{ABC} / \mathrm{DTG} / 3 \mathrm{TC} \\
(n=278)\end{array}$ & $\begin{array}{l}\mathrm{B} / \mathrm{F} / \mathrm{TAF} \\
(n=266)\end{array}$ & $\begin{array}{l}\text { ABC/DTG/3TC } \\
(n=266)\end{array}$ \\
\hline Fatigue/loss of energy & 38.6 & 35.6 & 30.7 & 35.7 & 35.7 & 36.5 & 34.6 & 38.3 \\
\hline Fevers/chills/sweats & 14.4 & 10.7 & 12.9 & 8.9 & 14.7 & 14.5 & 10.5 & 11.7 \\
\hline Dizzy/lightheadedness & 16.2 & 11.1 & 10.0 & 13.9 & 14.4 & 16.2 & 12.0 & 14.3 \\
\hline $\begin{array}{l}\text { Pain/numbness/tingling } \\
\text { in hands/feet }\end{array}$ & 24.1 & 24.3 & 17.8 & 20.4 & 20.3 & 23.2 & 21.9 & 25.9 \\
\hline Trouble remembering & 22.7 & 22.1 & 21.1 & 16.4 & 22.3 & 25.0 & 24.4 & 23.3 \\
\hline Nausea/vomiting & 7.2 & 8.2 & 6.4 & 7.5 & 4.0 & 7.9 & 4.5 & 8.6 \\
\hline Diarrhea/loose bowels & 16.5 & 15.7 & 15.0 & 16.8 & 15.8 & 16.3 & 11.7 & 13.9 \\
\hline $\mathrm{Sad} /$ down/depressed & 28.9 & 25.3 & 19.6 & 22.9 & 22.7 & 26.0 & 23.0 & 30.9 \\
\hline Nervous/anxious & 26.8 & 25.6 & 17.9 & 20.7 & 20.1 & 25.1 & 23.4 & 28.6 \\
\hline Difficulty sleeping & 37.1 & 35.5 & 32.7 & 33.9 & 31.3 & 38.8 & 32.0 & 35.3 \\
\hline $\begin{array}{l}\text { Skin problems/rash/ } \\
\text { itching }\end{array}$ & 21.2 & 15.7 & 14.6 & 14.6 & 17.7 & 20.3 & 22.6 & 21.5 \\
\hline $\begin{array}{l}\text { Coughing/trouble } \\
\text { breathing }\end{array}$ & 16.8 & 13.7 & 13.6 & 12.9 & 13.7 & 11.9 & 15.9 & 17.8 \\
\hline Headaches & 21.9 & 16.5 & 17.9 & 17.6 & 17.3 & 18.3 & 18.1 & 16.7 \\
\hline Loss of appetite & 10.4 & 6.5 & 6.8 & 8.6 & 5.4 & 10.9 & 6.4 & 8.3 \\
\hline $\begin{array}{l}\text { Bloating/pain/gas in } \\
\text { stomach }\end{array}$ & 22.7 & 23.5 & 20.8 & 24.4 & 17.3 & 21.9 & 19.3 & 19.4 \\
\hline Muscle aches/joint pain & 30.4 & 28.5 & 22.9 & 22.9 & 30.0 & 28.5 & 28.3 & 25.2 \\
\hline Problems with sex & 20.4 & 23.9 & 16.5 & 14.0 & 18.1 & 20.9 & 20.4 & 21.6 \\
\hline $\begin{array}{l}\text { Changes in body com- } \\
\text { position }\end{array}$ & 26.6 & 24.5 & 19.6 & 18.6 & 22.7 & 22.7 & 28.7 & 22.0 \\
\hline Weight loss/wasting & 12.2 & 7.9 & 7.2 & 5.7 & 11.2 & 9.0 & 10.6 & 6.4 \\
\hline Hair loss/changes & 7.9 & 6.9 & 7.5 & 3.2 & 4.3 & 6.5 & 9.4 & 9.1 \\
\hline
\end{tabular}

$n$ is the number of participants with response for at least one symptom

Bold characters are for significantly different percentages between treatment groups $(p<0.05), p$ value is calculated from the unadjusted logistic regression model

$A B C / D T G / 3 T C$ co-formulated abacavir, dolutegravir, and lamivudine, $B / F / T A F$ co-formulated bictegravir, emtricitabine, and tenofovir alafenamide

DTG/3TC groups over time, all favoring the B/F/TAF group. Three additional symptoms also showed that the effect over time was dependent on treatment group. These were headaches, bloating/pain/gas in stomach, and changes in body composition, each with lower prevalence over 48 weeks for $\mathrm{B} / \mathrm{F} / \mathrm{TAF}$. In virologically suppressed participants, the adjusted longitudinal models revealed a statistically significant difference between treatments over time (favoring $\mathrm{B} / \mathrm{F} /$ TAF over ABC/DTG/3TC) in the prevalence of six symptoms: dizzy/lightheadedness, nausea/vomiting, sad/down/ depressed, nervous/anxious, difficulty sleeping, and loss of appetite. No symptom favored the ABC/DTG/3TC group.

No covariate was significant in all symptom models; however, the presence of the bothersome symptoms at baseline, the HIV-SI symptom count at baseline, and SF-36 PCS score at baseline were significant for most symptom models. Each covariate was significant in at least one model. A complete table showing the coefficients, including findings for main effects and time by treatment interactions is provided in Table ESM2 of the Electronic Supplementary Material.

Tables 4 and 5 summarize the results for symptoms with statistically significant findings in the regression and/or longitudinal analyses. Fig. 1a and b show the observed prevalence of each symptom from Table 3 over time by treatment group that was statistically significantly different at two or more time points in the adjusted logistic regression model or at one time point in the adjusted logistic regression model and in the longitudinal model.

For treatment-naïve adults, the proportion of participants with poor sleep quality according to the PSQI was numerically less for B/F/TAF than AB/DTG/3TC at all postbaseline time points, with a significantly significant difference noted at week 12 (favoring B/F/TAF) in the adjusted logistic regression model. For virologically suppressed participants, 
Table 4 Summary of results from adjusted logistic regression analyses at weeks 4, 12, and 48 and longitudinal analyses in Study GS-US-3801489 (treatment-naïve)

\begin{tabular}{|c|c|c|c|c|c|}
\hline HIV-SI bothersome symptom & Week 4 & Week 12 & Week 48 & $\begin{array}{l}\text { Longi- } \\
\text { tudinal } \\
\text { model }\end{array}$ & Description of longitudinal findings \\
\hline Fatigue/loss of energy & $\checkmark$ & $\checkmark$ & $\checkmark$ & $\checkmark$ & $\begin{array}{l}\text { Decreased prevalence in } \mathrm{B} / \mathrm{F} / \mathrm{TAF} \text { group maintained over study } \\
\text { period without any changes in prevalence from week } 4 \text { to week } 48 \\
\text { for either group }\end{array}$ \\
\hline Dizzy/lightheadedness & $\checkmark$ & & $\checkmark$ & & $\begin{array}{l}\text { Decreased prevalence in } \mathrm{B} / \mathrm{F} / \mathrm{TAF} \text { group, } \mathrm{ABC} / \mathrm{DTG} / 3 \mathrm{TC} \text { group with } \\
\text { fluctuating prevalence }\end{array}$ \\
\hline Nausea/vomiting & $\checkmark$ & $\checkmark$ & & $\checkmark$ & $\begin{array}{l}\text { Increased initial prevalence in } \mathrm{ABC} / \mathrm{DTG} / 3 \mathrm{TC} \text { group (at week } 4 \text { ) } \\
\text { maintained over study period with decreasing prevalence in both } \\
\text { groups from week } 4 \text { to week } 48\end{array}$ \\
\hline Difficulty sleeping & & $\checkmark$ & $\checkmark$ & & $\begin{array}{l}\text { No differences in prevalence observed between groups from week } 4 \\
\text { to week } 48\end{array}$ \\
\hline Headaches & & & $\checkmark$ & $\ddagger$ & Decreased prevalence in $\mathrm{B} / \mathrm{F} / \mathrm{TAF}$ group from week 4 to week 48 \\
\hline Loss of appetite & & $\checkmark$ & & $\checkmark$ & $\begin{array}{l}\text { Decreased prevalence in } \mathrm{B} / \mathrm{F} / \mathrm{TAF} \text { group, } \mathrm{ABC} / \mathrm{DTG} / 3 \mathrm{TC} \text { group with } \\
\text { fluctuating prevalence }\end{array}$ \\
\hline Bloating/pain/gas in stomach & & & $\checkmark$ & $\ddagger$ & $\begin{array}{l}\text { No differences in prevalence observed between groups from week } 4 \\
\text { to week } 48\end{array}$ \\
\hline Changes in body composition & & & & $\ddagger$ & $\begin{array}{l}\text { Slight increased prevalence in } \mathrm{B} / \mathrm{F} / \mathrm{TAF} \text { group and a greater } \\
\text { increased prevalence in } \mathrm{ABC} / \mathrm{DTG} / 3 \mathrm{TC} \text { group }\end{array}$ \\
\hline Weight loss/wasting & & $\checkmark$ & & & $\begin{array}{l}\text { No differences in prevalence observed between groups from week } 4 \\
\text { to week } 48\end{array}$ \\
\hline Poor sleep quality (from PSQI) & & $\checkmark$ & & & $\begin{array}{l}\text { Decreased prevalence in } \mathrm{B} / \mathrm{F} / \mathrm{TAF} \text { group starting from week } 4 \text { and } \\
\text { maintained over study period with minimal changes in } \mathrm{ABC} / \\
\text { DTG/3TC group }\end{array}$ \\
\hline
\end{tabular}

$A B C / D T G / 3 T C$ co-formulated abacavir, dolutegravir, and lamivudine, $B / F / T A F$ co-formulated bictegravir, emtricitabine, and tenofovir alafenamide, HIV-SI HIV Symptom Index, PSQI Pittsburgh Sleep Quality Index

$\checkmark=$ Statistically significant favoring the B/F/TAF group; $\neq=$ statistically significant time-by-treatment interaction (i.e., changes over time depend on treatment)

a significant treatment difference was noted at weeks 4 and 12 in the adjusted logistic regression model as well as in the longitudinal model (all favoring B/F/TAF). Results from the PSQI are shown in Tables 4 and 5 as well as plotted in Fig. $1 b$.

\section{Discussion}

Our analyses represent the first prospective, randomized, double-blind comparison of patient-reported symptoms among HIV-1-infected adults who were either treatment naïve or virologically suppressed and randomized to receive $\mathrm{B} / \mathrm{F} / \mathrm{TAF}$ versus $\mathrm{ABC} / \mathrm{DTG} / 3 \mathrm{TC}$. The results indicate that $\mathrm{B} / \mathrm{F} / \mathrm{TAF}$ was associated with fewer patient-reported bothersome symptoms over 48 weeks, including nausea and some neuropsychiatric symptoms.

Descriptive analyses showed that differences between $\mathrm{B} / \mathrm{F} / \mathrm{TAF}$ and ABC/DTG/3TC appeared as early as week 4 and were generally maintained through 48 weeks. In treatment-naïve participants, the greatest differences between the two treatment groups were seen in reports of nausea/ vomiting, difficulty sleeping, fatigue/loss of energy, and dizzy/lightheadedness (Table 4). In virologically suppressed adults, nausea/vomiting and difficulty sleeping were again observed to be reported by a significantly greater proportion of those receiving ABC/DTG/3TC, as were the symptoms of feeling sad/down/depressed and nervous/anxious (Table 5). Sleep findings on the HIV-SI were supported by findings of poor sleep quality on the PSQI. Both unadjusted/ adjusted and longitudinal analyses were reported here, since unadjusted/adjusted logistic regression models at fixed time points are useful for assessing treatment differences at the first postbaseline visit (week 4) and at the primary analysis time point (week 48), while the longitudinal model aims to assess treatment differences over time. This provides a collective assessment of all of the time points, using the individual's responses at all time points (and the associated variability) to provide a singular assessment. The longitudinal analysis has the advantage of distilling the numerous time points into a single value, albeit from a more complicated model. As both the fixed time point and the longitudinal analyses seek to answer different (but related) questions, they both provide insights into the data. As shown in 
Table 5 Summary of results from adjusted logistic regression analyses at weeks 4, 12, and 48 and longitudinal analyses in Study GS-US-3801844 (HIV-1 suppressed)

\begin{tabular}{|c|c|c|c|c|c|}
\hline HIV-SI bothersome symptom & Week 4 & Week 12 & Week 48 & $\begin{array}{l}\text { Longi- } \\
\text { tudinal } \\
\text { model }\end{array}$ & Description of longitudinal findings \\
\hline Fatigue/loss of energy & $\checkmark$ & & & & $\begin{array}{l}\text { No differences in prevalence observed between groups from } \\
\text { week } 4 \text { to week } 48\end{array}$ \\
\hline Dizzy/lightheadedness & $\checkmark$ & & & $\checkmark$ & $\begin{array}{l}\text { Decreased prevalence in } \mathrm{B} / \mathrm{FTC} / \mathrm{TAF} \text { group at week } 4 \text { sustained } \\
\text { through week } 48 \text {, some increase in prevalence for } \mathrm{ABC} / \\
\text { DTG/3TC group from week } 4 \text { to week } 48\end{array}$ \\
\hline Pain/numbness/tingling in hands/feet & & & $\checkmark$ & & $\begin{array}{l}\text { No differences in prevalence observed between groups from } \\
\text { week } 4 \text { to week } 48\end{array}$ \\
\hline Nausea/vomiting & & $\checkmark$ & $\checkmark$ & $\checkmark$ & $\begin{array}{l}\text { Decreased prevalence in B/F/TAF group starting from week } 4 \\
\text { and maintained over study period with minimal changes in } \\
\text { ABC/DTG/3TC group }\end{array}$ \\
\hline $\mathrm{Sad} /$ down/depressed & $\checkmark$ & & $\checkmark$ & $\checkmark$ & $\begin{array}{l}\text { Decreased prevalence in B/F/TAF group starting from week } 4 \\
\text { and maintained over study period vs increased prevalence in } \\
\text { ABC/DTG/3TC from week } 4 \text { to week } 48\end{array}$ \\
\hline Nervous/anxious & $\checkmark$ & $\checkmark$ & $\checkmark$ & $\checkmark$ & $\begin{array}{l}\text { Decreased prevalence starting from week } 4 \text { and maintained } \\
\text { over study period in B/F/TAF group vs fluctuating prevalence } \\
\text { in ABC/DTG/3TC group }\end{array}$ \\
\hline Difficulty sleeping & & $\checkmark$ & & $\checkmark$ & $\begin{array}{l}\text { Decreased prevalence in } \mathrm{B} / \mathrm{F} / \mathrm{TAF} \text { group maintained over study } \\
\text { period vs fluctuating prevalence in } \mathrm{ABC} / \mathrm{DTG} / 3 \mathrm{TC} \text { group }\end{array}$ \\
\hline Loss of appetite & & $\checkmark$ & & $\checkmark$ & $\begin{array}{l}\text { Decreased prevalence starting from week } 4 \text { in } \mathrm{B} / \mathrm{F} / \mathrm{TAF} \text { group } \\
\text { maintained over study period vs fluctuating prevalence in } \\
\text { ABC/DTG/3TC group }\end{array}$ \\
\hline Bloating/pain/gas in stomach & & $\checkmark$ & & & $\begin{array}{l}\text { No differences in prevalence observed between groups from } \\
\text { week } 4 \text { to week } 48\end{array}$ \\
\hline Hair loss/changes & $x$ & & & & $\begin{array}{l}\text { No differences in prevalence observed between groups from } \\
\text { week } 4 \text { to week } 48\end{array}$ \\
\hline Poor sleep quality (from PSQI) & $\checkmark$ & $\checkmark$ & & $\checkmark$ & $\begin{array}{l}\text { Decreased prevalence in B/F/TAF group starting from week } 4 \\
\text { and maintained over study period with minimal changes in } \\
\text { ABC/DTG/3TC group }\end{array}$ \\
\hline
\end{tabular}

$A B C / D T G / 3 T C$ co-formulated abacavir, dolutegravir, and lamivudine, $B / F / T A F$ co-formulated bictegravir, emtricitabine, and tenofovir alafenamide, HIV-SI HIV Symptom Index, PSQI Pittsburgh Sleep Quality Index

$\checkmark=$ Statistically significant favoring the B/F/TAF group; $x=$ statistically significant favoring the ABC/DTG/3TC group

Tables 4 and 5, some symptoms may be significant using fixed time-point models, but not in the longitudinal model. This is expected as the longitudinal model looks across all time points. A marginal finding at a single time point may not be significant when considering all time points. Importantly, no models showed significant treatment differences in the longitudinal model without at least one fixed time-point model showing significance as well. However, it should be noted that there were no large difference in PCS and MCS, aggregate measures from the SF-36, over time for either regimen.

The use of PRO tools within these studies provides insight into patient-reported symptoms that may be underreported by individuals during standard screening for adverse drug events [38]. Others have suggested increasing the use of PRO tools in clinical research in order to differentiate in greater detail the benefit of various regimens [31, 39, 40]. Additionally, the use of longitudinal modeling allows for a greater understanding of the prevalence of HIV symptoms over time.

As the efficacy of well tolerated antiretroviral FDC regimens in clinical trials reaches above $90 \%$, it is important to demonstrate the comparative impact of various FDC options on HRQL measures and the degree to which they are perceived by the individual as being bothersome. Tolerability and acceptance of long-term medication affect adherence to and persistence with that therapy. Adherence to antiretroviral treatments has been shown to improve outcomes and lower healthcare costs. Results from a real-world database analysis found that individuals who were $95 \%$ or more adherent to therapy had lower hospitalization rates and associated healthcare costs compared with those who had lower adherence rates [41]. Similarly, increased cost has been associated with adverse events. Among 11 adverse events (rash, nausea or vomiting, diarrhea, dizziness, headache, sleep-related symptoms, hepatotoxicity, lipid disorders, 

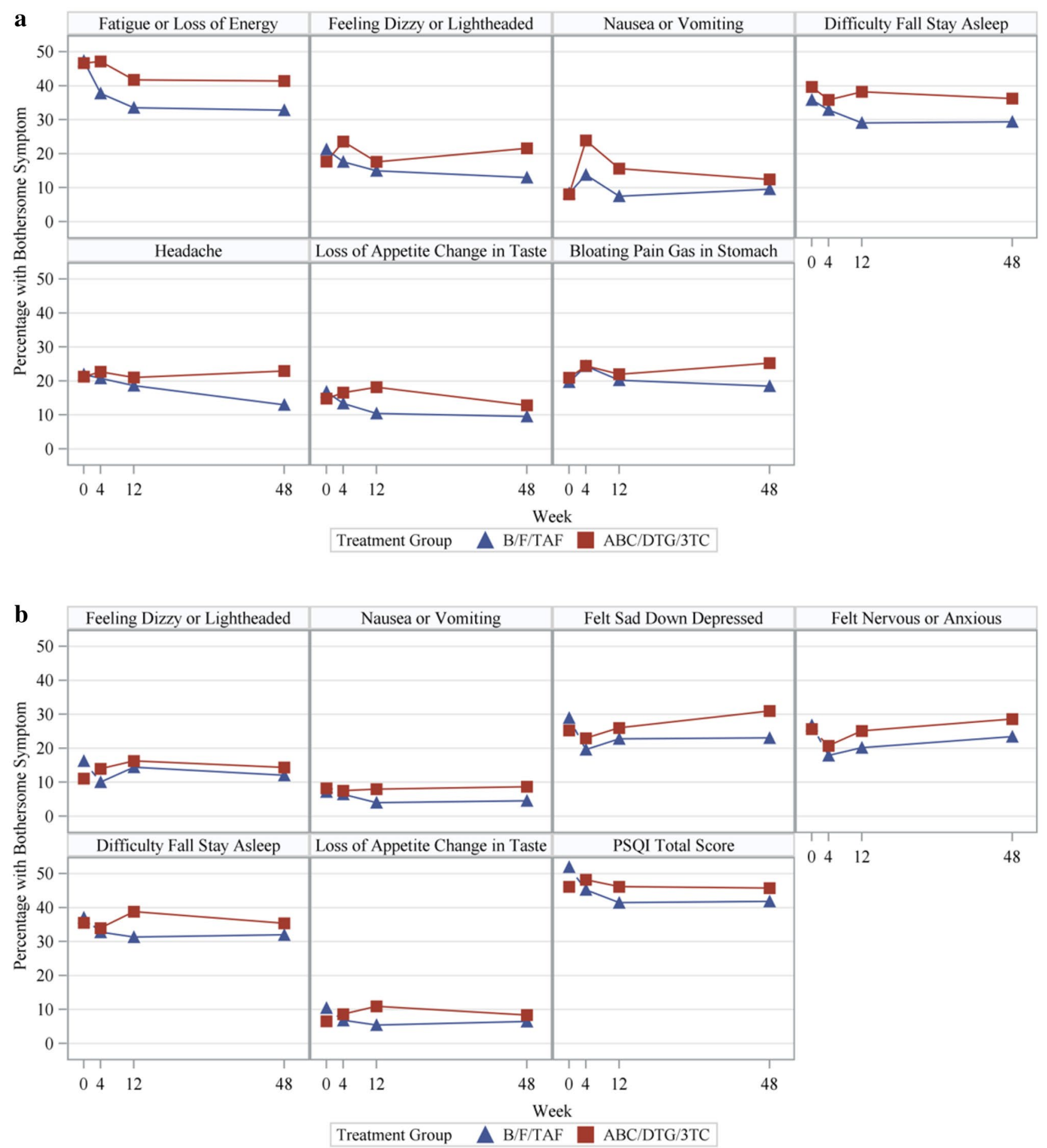

Fig. 1 a Prevalence of bothersome symptoms over time by treatment group in Study GS-US-380-1489 (Treatment-Naïve). b Prevalence of bothersome symptoms over time by treatment group in Study GS-US-380-1844 (HIV-1 Suppressed)

depression, anxiety, and suicide or self-injury) observed with NRTI use, sleep-related adverse events had the highest outpatient care cost, with a mean per-episode cost of US\$6438 (IQR 615-5882; median US\$1785). Over a 5-year time period, sleep-related adverse events accounted for the second highest overall mean healthcare cost of US $\$ 8307$, with only nausea and vomiting exceeding it with US $\$ 12,833$ [42].

There are limitations to this analysis that should be considered. While the results of the two studies are generalizable 
to a broad patient population as both treatment-naïve and virologically suppressed adults were evaluated, they may not be generalizable to those who are not White males as the majority of the study participants were male and White. Furthermore, unlike investigator-reported symptoms, those reported by study participants are not graded using a standard scheme. Therefore, the severity of participant-reported symptoms may not be clear. However, the Likert scale did provide a sense of the degree to which a symptom was considered bothersome. Arguably, any symptom perceived to be bothersome to any extent is undesirable. Lastly, generalizability of study findings must be done with caution as the current study populations were relatively healthy with few co-morbidities; those who switched to B/F/TAF were stably suppressed with no tolerability issues to their antiretroviral therapy at baseline.

\section{Conclusions}

Overall, these studies demonstrated that over approximately 1 year of follow-up after initiating or switching antiretroviral treatments, B/F/TAF was associated with fewer bothersome symptoms, especially nausea and vomiting, as well as sleep difficulties, than with ABC/DTG/3TC. Patient-reported symptoms may be an important consideration when selecting among highly efficacious options for the treatment of HIV infection and should continue to be studied in clinical trials.

Data Availability Statement The datasets generated during and/or analyzed during the current study are not publicly available due to the fact that they are proprietary intellectual property of the Sponsor (Gilead Sciences), but are available from the corresponding author on reasonable request.

\begin{abstract}
Acknowledgements Dr. Wohl contributed to the acquisition of the data and manuscript preparation. Dr. Clarke contributed to the acquisition of the data and manuscript preparation. Dr. Maggiolo contributed to the acquisition of the data and manuscript preparation. Dr. Garner contributed to the acquisition of data, analysis and interpretation of data, and manuscript preparation. Dr. Laouri contributed to the interpretation of data and the manuscript preparation. Dr. Martin contributed to the acquisition of the data and manuscript preparation. Dr. Quirk contributed to the acquisition of the data and manuscript preparation. We would like to thank Dr. Xuelian Wei and Dr. Hui Liu (both Gilead) for providing additional data analyses and interpretation, and Anna Kido (Gilead) for providing editorial assistance.
\end{abstract}

\section{Compliance with Ethical Standards}

Potential Conflicts of Interest and Sources of Funding: Dr Wohl has served on advisory boards for Gilead and Janssen, and has received research grants to the University of North Carolina from Gilead. Dr Clarke has served on advisory boards for GSK and Gilead, and has received conference attendance support from Janssen, BMS and Gilead, and received a speaker fee from Gilead. Dr Maggiolo has served on advisory boards for Abbvie, Bristol-Myers Squibb, Gilead, GlaxoSmithKline, Tibotec, and has received research grants to ASST Papa Giovanni XXIII from Abbvie, Bristol-Myers Squibb, Gilead, GlaxoSmithKline, and Janssen. Drs Garner, Laouri, Martin, and Quirk are employees of Gilead Sciences and hold stock options in the company. This study was funded by Gilead Sciences. This study was conducted in accordance with the Declaration of Helsinki. The protocol was reviewed and approved by central or site-specific institutional review boards or ethics committees covering all participating sites. All participants provided written informed consent.

Open Access This article is distributed under the terms of the Creative Commons Attribution-NonCommercial 4.0 International License (http://creativecommons.org/licenses/by-nc/4.0/), which permits any noncommercial use, distribution, and reproduction in any medium, provided you give appropriate credit to the original author(s) and the source, provide a link to the Creative Commons license, and indicate if changes were made.

\section{References}

1. Gunthard HF, Saag MS, Benson CA, del Rio C, Eron JJ, Gallant JE, et al. Antiretroviral drugs for treatment and prevention of HIV infection in adults: 2016 recommendations of the international antiviral society-USA panel. JAMA. 2016;316:191-210.

2. European AIDS Clinical Society. Guidelines. Version 9.0. October 2017. Brussels: European AIDS Clinical Society, 2017. www. eacsociety.org/files/guidelines_9.0-english.pdf. Accessed 30 May 2018.

3. Panel on Antiretroviral Guidelines for Adults and Adolescents. Guidelines for the use of antiretroviral agents in HIV-1-infected adults and adolescents: U. S. Department of Health and Human Services, Washington D.C., USA. https://aidsinfo.nih.gov/conte ntfiles/lvguidelines/adultandadolescentgl.pdf. Last Updated 27 March 2018. Accessed 30 May 2018.

4. Blanco J, Montaner JSG, Matconi VC, et al. Lower prevalence of drug resistance mutations at first-line virological failure to firstline therapy with atripla vs. tenpfovir + emtricitabine/lamivudine + efavirenz administered on a multiple tablet therapy. AIDS. 2015;28:2531-9.

5. Elliot E, Chirwa M, Boffito M. How recent findings on the pharmacokinetics and pharmacodynamics of integrase inhibitors can inform clinical use. Curr Opin Infect Dis. 2017;30:58-73.

6. Sax PE, Tierney C, Collier AC, et al. Abacavir/lamivudine versus tenofovir/emtricitabine as part of combination regimens for initial treatment of HIV: final results. J Infect Dis. 2011;204:1191-201.

7. Quercia R, Roberts J, Murungi A, Curtis L, Payvandi N, Koteff J, Aboud M. Psychiatric adverse events from the DTG ART-naive phase III/IIIb clinical trials. HIV Glasgow 2016 Poster P210.

8. Hsu R, Fusco J, Henegar C, Carpio F, Mounzer K, Wohlfeiler M, Vannappagari V, Aboud M, Curtis L, Fusco G. Psychiatric disorders observed in HIV + patients using 6 common 3rd agents in OPERA. In: Conference on Retroviruses and Opportunistic Infections CROI. Seattle 2017 Feb 13. http://www.natap.org/2017/ CROI/croi_62.htm. Accessed 25 Oct 2017.

9. Hoffmann C, Welz T, Sabranski M, Kolb M, Wolf E, Stellbrink HJ, Wyen C. Higher rates of neuropsychiatric adverse events leading to dolutegravir discontinuation in women and older patients. HIV Med. 2017;18:56-63.

10. Llibre JM, Esteve A, Miro JP, Mateo G, Curran A, Podzamczer D. Discontinuation of DTG, EVG/c, and RAL due to toxicity in 
a prospective cohort. In: Conference on retroviruses and opportunistic infections CROI. Seattle 2017 Feb 13. http://www.natap .org/2017/CROI/croi_59.htm. Accessed 25 Oct 2017.

11. Yagura H, Watanabe D, Nakauchi T, Tomishima K, Kasai D, Nishida Y. Effect of Dolutegravir plasma concentration on central nervous system side effect. In: Conference on retroviruses and opportunistic infections CROI. Seattle 2017. http://www.natap .org/2017/CROI/croi_85.htm. Accessed 25 Oct 2017.

12. Viswanathan P, Baro E, Soon G, Sherwat A, Struble K. Neuropsychiatric adverse events associated with integrase strand transfer inhibitors. In: Conference on retroviruses and opportunistic infections CROI. Seattle 2017. http://www.natap.org/2017/CROI/ croi_60.htm. Accessed 25 Oct 2017.

13. Wohl D, Mills A, Mera R, Pionkowsky D. Selected CNS outcomes among INSTI antiretrovirals. ID Week 2017 Abstract \#1687.

14. Tsiang M, Jones GS, Goldsmith J, et al. Antiviral activity of bictegravir (GS-9883), a novel potent HIV-1 integrase strand transfer inhibitor with an improved resistance profile. Antimicrob Agents Chemother. 2016;60:7086-97.

15. Gallant J, Lazzarin A, Mills A, et al. Bictegravir, emtricitabine, and tenofovir alafenamide versus dolutegravir, abacavir, and lamivudine for initial treatment of HIV-1 infection (GS-US-380-1489): a double-blind, multicentre, phase 3, randomised controlled noninferiority trial. Lancet. 2017;390:2063-72.

16. Sax PE, Pozniak A, Montes ML, et al. Coformulated bictegravir, emtricitabine, and tenofovir alafenamide versus dolutegravir with emtricitabine and tenofovir alafenamide, for initial treatment of HIV-1 infection (GS-US-380-1490): a randomised, double-blind, multicentre, phase 3, non-inferiority trial. Lancet. 2017;S0140-6736(17):32340-1.

17. Molina J-M, Ward DA, Brar I, et al. Switching to fixed-dose bictegravir, emtricitabine, and tenofovir alafenamide from dolutegravir plus abacavir and lamivudine in virologically suppressed HIV-1 infected adults: a randomised, double-blinded, multicentre, activecontrolled, phase 3, non-inferiority trial. Lancet HIV. 2018. https ://doi.org/10.1016/S2352-3018(18)30092-4.

18. Daar ES, DeJesus E, Ruane P, et al. Switching to fixed-dose bictegravir, emtricitabine, and tenofovir alafenamide from boosted protease inhibitor-based regimens in virologically suppressed HIV-1 infected adults: a randomised, open-label, multicentre, active-controlled, phase 3, non-inferiority trial. Lancet HIV. 2018. (In press).

19. Elion RA, Althoff KN, Zhang J, et al. Recent abacavir use increases risk of type 1 and type 2 myocardial infarctions among adults with HIV. J Acquir Immune Defic Syndr. 2018;78:62-72.

20. Marcus JL, Neugebauer RS, Leyden WA, et al. Use of abacavir and risk of cardiovascular disease among HIV-infected individuals. J Acquir Immune Defic Syndr. 2016;71:413-9.

21. Sabin CA, Reiss P, Ryom L. D:A:D Study Group. Is there continued evidence for an association between abacavir usage and myocardial infarction risk in individuals with HIV? a cohort collaboration. BMC Med. 2016;14:61.

22. de Boer MG, van den Ber GE, van Holten N, et al. Intolerance of dolutegravir-containing combination antiretroviral therapy regimens in real-life clinical practice. AIDS. 2016;30:2831-4.

23. Fettiplace A, Stainsby C, Winston A, et al. Psychiatric symptoms in patients receiving dolutegravir. J Acquir Defic Syndr. 2017;74:423-31.

24. Chan HLY, Fung S, Seto WK, et al. Tenofovir alafenamide versus tenofovir disoproxil fumarate for the treatment of $\mathrm{HBeAg}$-positive chronic hepatitis B virus infection: a randomised, double-blind, phase 3, non-inferiority trial. Lancet Gastroenterol Hepatol. 2016;1:185-95.

25. Scott LJ, Chan HLY. Tenofovir alafenamide: a review in chronic hepatitis B. Drugs. 2017;77:1017-28.

26. European Medicines Agency. Triumeq (dolutegravir, abacavir, lamivudine). Summary of product characteristics. 2018. http:// www.ema.europa.eu/docs/en_GB/document_library/EPAR_Product_Information/human/002754/WC500175596.pdf. Accessed 30 Mar 2018.

27. Wu AW. Quality of life assessment comes of age in the era of highly active antiretroviral therapy. AIDS. 2000;14:1449-51.

28. Justice AC, Modur SP, Tate JP, et al. Predictive accuracy of the veterans aging cohort study index for mortality with HIV infection: a North American cross cohort analysis. J Acquir Immune Defic Syndr. 2013;62:149-63.

29. Sterling RK, Lissen E, Clumeck N, et al. Development of a simple noninvasive index to predict significant fibrosis in patients with HIV/HCV coinfection. Hepatology. 2006;43:1317-25.

30. Justice A, Holmes W, Gifford A, et al. Development and validation of a self-completed HIV symptom index. J Clin Epidemiol. 2001;2001(54):S77-90.

31. Simpson KN, Hanson KA, Harding G, Haider S, Tawadrous M, Khachatryan A, et al. Patient reported outcome instruments used in clinical trials of HIV-infected adults on NNRTI-based therapy: a 10-year review. Health Qual Life Outcomes. 2013;11:164.

32. Edelman EJ, Gordon K, Rodriguez-Barradas MC, Justice AC, Vacs Project T. Patient-reported symptoms on the antiretroviral regimen efavirenz/emtricitabine/tenofovir. AIDS Patient Care STDS. 2012;26:312-9.

33. Brazier JE, Harper R, Jones NM, et al. Validating the SF-36 health survey questionnaire: new outcome measure for primary care. BMJ. 1992;305:160-4.

34. Ware JE Jr, Sherbourne CD. The MOS 36-item short-form health survey (SF-36). I. Conceptual framework and item selection. Med Care. 1992;30:473-83.

35. Hsiung PC, Fang CT, Chang YY, Chen MY, Wang JD. Comparison of WHOQOL-bREF and SF-36 in patients with HIV infection. Qual Life Res. 2005;14:141-50.

36. Buysse DJ, Reynolds CF III, Monk TH, Berman SR, Kupfer DJ. The Pittsburgh Sleep Quality Index: a new instrument for psychiatric practice and research. Psychiatry Res. 1989;28:193-213.

37. Reilly MC, Zbrozek AS, Dukes EM. The validity and reproducibility of a work productivity and activity impairment instrument. Pharmacoeconomics. 1993;4:353-65.

38. Edelman EJ, Gordon K, Justice AC. Patient and provider-reported symptoms in the post-cART era. AIDS Behav. 2011;15:853-61.

39. Kozak MS, Mugavero MJ, Ye J, et al. Patient reported outcomes in routine care: advancing data capture for HIV cohort research. Clin Infect Dis. 2012;2012(54):141-7.

40. Engler K, Lessard D, Lebouche B. A review of HIV-specific patient-reported outcome measures. Patient. 2017;10:187-202.

41. Cohen CJ, Meyers JL, Davis KL. Association between daily antiretroviral pill burden and treatment adherence, hospitalisation risk, and other healthcare utilisation and costs in a US medicaid population with HIV. BMJ Open. 2013;3:e003028.

42. Boulanger L, Chambers R, Nedrow K, et al. Costs of adverse events among patients with HIV infection treated with nonnucleoside reverse transcriptase inhibitors. HIV Med. 2014;15:488-98. 


\section{Affiliations}

David Wohl ${ }^{1} \cdot$ Amanda Clarke $^{2} \cdot$ Franco Maggiolo $^{3} \cdot$ Will Garner $^{4} \cdot$ Marianne Laouri $^{4} \cdot$ Hal Martin $^{4} \cdot$ Erin Quirk $^{4}$

$\triangle$ Hal Martin

Hal.Martin@gilead.com

1 University of North Carolina at Chapel Hill, Chapel Hill, NC, USA

2 Royal Sussex County Hospital, Brighton, UK
ASST Papa Giovanni XXIII, Bergamo, Italy

4 Gilead Sciences, Inc, 333 Lakeside Drive, Foster City, CA 94404, USA 